\title{
The Profile of Ciprofloxacin-Resistant Bacteria in the Rectum of Patients Undergoing Transrectal Ultrasound-Guided Prostate Biopsy, Its Relation to Post-Biopsy Infection and Alternative Prophylactic Antibiotics
}

\author{
Tonny Loho* ${ }^{1}$, Agus Rizal A. H. Hamid ${ }^{2}$, Rainy Umbas ${ }^{2}, \mathrm{Na}^{\prime i m a t u l ~ M a h a n a n i}{ }^{1}$ \\ ${ }^{1}$ Department of Clinical Pathology, Faculty of Medicine, Universitas Indonesia, Jakarta \\ ${ }^{2}$ Department of Urology, Faculty of Medicine, Universitas Indonesia, Jakarta
}

\section{ARTICLE INFO}

Received : 05 July 2020

Reviewed : 24 July 2020

Accepted : 03 November 2020

Keywords:

ultrasonography, biopsy, prostatic hyperplasia, drug resistance, antibiotic

\author{
ABSTRACT
}

Background: Fluoroquinolone has been routinely given as a prophylactic antibiotic to patients undergoing transrectal ultrasound (TRUS)-guided prostate biopsy. Currently, there is no data on the profile of fluoroquinolone-resistant bacteria, its association with post-biopsy sepsis, and alternative prophylactic antibiotics.

\begin{abstract}
Methods: A cross-sectional study was conducted in patients undergoing TRUS-guided prostate biopsy at Urology Clinic, Cipto Mangunkusumo National Central General Hospital between August and December 2015. Specimens were taken from rectal swabs prior to biopsy. Gramnegative bacilli and Gram-positive cocci were characterized on the Vitek ${ }^{\circledR} 2$ using GN and GP cards (BioMérieux, USA). Antimicrobial susceptibility testing was done by Kirby Bauer disc diffusion method. Post-biopsy infection was monitored within one week after biopsy by telephone call and diagnosed by clinical examination.
\end{abstract}

Results: A total of 52 patients aged 52-80 years were enrolled. Sixty-six isolates grew from 52 swabs; 14 swabs among them grew 2 isolates. The commonest pathogen was Escherichia coli (78.8\%), followed by Klebsiella pneumoniae (13.6\%), Enterococcus faecium (3.0\%), Acinetobacter haemolyticus, Morganella morganii subsp. morganii, and Enterococcus faecalis (1.5\%), respectively. Ciprofloxacin-resistant bacteria were found in $33(50.0 \%)$ isolates which were predominated by E. coli. Meanwhile, $90.4 \%$ and $96.2 \%$ of isolates were sensitive to amoxicillinclavulanate and ampicillin-sulbactam, respectively. Post-biopsy infection was established in 7 patients requiring no hospitalization.

*Corresponding author:

Tonny Loho

Department of Clinical Pathology,

Faculty of Medicine, Universitas

Indonesia, Jakarta

tonnyloho@yahoo.com
Conclusions: The proportion of ciprofloxacin-resistant bacteria in the rectum among patients undergoing prostate biopsy was $50.0 \%$. Escherichia coli is the commonest resistant pathogen to fluoroquinolone. The recommended alternatives for prophylactic antibiotics are amoxicillinclavulanate and ampicillin-sulbactam. Routine pre-biopsy rectal swab cultures should also be encouraged.

\section{INTRODUCTION}

Transrectal ultrasound (TRUS)-guided prostate biopsy is the standard technique for obtaining a histological diagnosis of prostatic carcinoma [1]. The commonest indications for TRUS biopsy are a raised prostate specific antigen (PSA) level and/or an abnormal digital rectal examination. According to recent estimates, approximately one million TRUS biopsies are performed annually in the United States [2]. Although generally considered as a safe and well-tolerated procedure, post-biopsy complications are reported in up to $50 \%$ of cases, which include pain, hematuria, hematospermia, urinary retention, and infection [3].

A variety of infectious complications may occur following TRUS biopsy, ranging from asymptomatic bacteriuria or urinary tract infection (UTI) to prostatitis, bacteremia, and severe sepsis [3]. Acute prostatitis was reported to occur in $1.3 \%$ of patients after the first biopsy and $6.8 \%$ after repeated biopsy [4]. The reported incidence of UTI after TRUS biopsy typically ranges between $2 \%$ and $6 \%$ with approximately $30 \%-50 \%$ of these patients having accompanying bacteremia $[5,6]$. Bacteremia is frequently accompanied by severe sepsis, 
which has an overall incidence of $0.1 \%-2.2 \%$ following TRUS biopsy [3].

The primary mechanism of post-TRUS biopsy sepsis is likely to be direct inoculation of bacteria from the rectal mucosa by the biopsy needle into the prostate, blood vessels, or urinary tract. This is supported by high reported rates of bacteremia (16\%-75\%) and bacteriuria (36\%-53\%) immediately post-procedure in the absence of prophylactic antibiotics and the fact that most infections manifest clinically within 3 days of TRUS biopsy [7]. In addition, pre-existing infection or inflammation may also contribute to post-biopsy infections although the value of routine urine culture and pre-biopsy treatment of asymptomatic bacteriuria remains controversial [3].

Strong evidence exists to support the use of prophylactic antibiotic prior to TRUS biopsy [8,9]. Fluoroquinolones are still the most frequently used antibiotic for prophylaxis in TRUS-guided prostate biopsy [7]. Prophylactic antibiotic with fluoroquinolones is recommended in several international guidelines to prevent infections in patients undergoing TRUS-guided prostate biopsy $[10,11]$. They are particularly useful due to their broad spectrum of activity against intestinal flora and high prostatic tissue levels obtained after oral administration [12].

Cipto Mangunkusumo National Central General Hospital (CMNCGH) is one of the top referral hospitals in Jakarta with 990 beds, which makes it the largest general and teaching hospital in Indonesia. Infection due to resistant bacteria to ciprofloxacin was recently suspected due to the increasing number of hospitalized cases with post-biopsy infection. However, the profile of fluoroquinolone-resistant bacteria in the rectum at the time of biopsy is not known. Therefore, this study was aimed to obtain the bacterial and antibiogram profile of rectal swabs among patients undergoing TRUSguided prostate biopsy, the following incidence of postbiopsy infections at CMNCGH, and alternative prophylactic antibiotics.

\section{METHODS}

\section{Study Design and Subjects}

This was a cross-sectional study in male patients undergoing TRUS-guided prostate biopsy at the Urology Department between August and December 2015. Patients were included if they were willing to undergo a rectal swab prior to a prostate biopsy. All patients gave their written consent before enrolment. Ethical approval letter number 766/UN2.F1/ETIK/2015 was issued by the Ethical Committee for Medical Research, Faculty of Medicine, Universitas Indonesia. The minimum sample size was calculated by estimating a population proportion with specified absolute precision [13]. The anticipated population proportion was $14 \%$. The required sample size at $95 \%$ confidence level and $10 \%$ absolute precision were 47 . Clinical assessment was done on each patient, and the patients' data were collected from their medical records including current urinary tract catheterization, the presence of diabetes mellitus, the history of UTI, hospital admission, fluoroquinolone, and other antibiotic treatments received in the past 6 months, and previous prostate biopsy.

\section{Rectal Swab Procedure and Microorganism Culture}

The prophylactic antibiotic ciprofloxacin $500 \mathrm{mg}$ tablet was given 1 hour before biopsy and then, after biopsy, the patient was given a prescription for another 5 tablets of ciprofloxacin $500 \mathrm{mg}$ to be taken every 12 hours. About one hour before TRUS-guided prostate biopsy, the urologist obtained a rectal swab by using a sterile swab inserted into the rectum. The swab was then put into the Stuart transport medium and was brought to the laboratory in less than one hour. In the laboratory, the swab was streaked onto sheep blood agar and MacConkey agar and then dipped into the thioglycolate broth. After overnight incubation at $35^{\circ} \mathrm{C}$ in ambient air, a single grown colony was identified with VITEK® 2 GN for Gramnegative bacilli and VITEK® 2 GP for Gram-positive cocci. VITEK $尺 2$ Gram-negative bacilli identification was controlled with Stenotrophomonas maltophilia ATCC 17666 and Gram-positive cocci were controlled with Enterococcus casseliflavus ATCC 700327. Antibiotic susceptibility testing was done by disc diffusion method according to Kirby Bauer. Escherichia coli ATCC 25922, Staphylococcus aureus ATCC 25923, and Pseudomonas aeruginosa ATCC 27853 were used as quality control bacteria for antibiotic susceptibility testing. The interpretation of susceptibility testing was based on the 2014 Clinical Laboratory Standards Institute guidelines.

The susceptibility testing included 17 antibiotics, i.e. ciprofloxacin, levofloxacin, gentamicin, amikacin, ampicillin-sulbactam, cephalothin, cefotaxime, amoxicillinclavulanate, ceftriaxone, ceftazidime, cefoperazone, piperacillin-tazobactam, cefepime, imipenem, doripenem, meropenem, and ertapenem. The panel test for Enterococcus faecium and Enterofoccus faecalis consisted of 7 antibiotics, i.e. ciprofloxacin, levofloxacin, ampicillin, teicoplanin, linezolid, vancomycin, and fosfomycin.

\section{Post-Biopsy Assessment}

After the biopsy procedure, patients were discharged and then monitored by phone on day- 3 and day- 7 . They were asked whether they had a fever and/or pain after being biopsied. If there were a fever and pain, they were asked to come to the outpatient urology clinic for further assessment. Post-biopsy infection was diagnosed by the urologist who did the biopsy. 


\section{Statistical analysis}

The characteristics of the study subjects, distribution of isolated pathogens, and antibiogram data were presented descriptively. The relationship between risk factors and post-biopsy infection was tested using Fisher's exact test. A $P$ value of less than 0.05 was considered significant. Statistical analysis was performed using SPSS software version 17.0 for Windows PC (SPSS Inc., Chicago, IL, USA).

\section{RESULTS}

\section{Demography}

\section{Patients' characteristics}

A total of 52 male patients were enrolled in this study. Patients' mean age was $67.2+7.8$ years, ranging from 52 to 80 years. Most patients (96.2\%) received outpatient care. Other characteristics are shown in Table 1. Sixtysix bacterial isolates grew from the 52 rectal swabs obtained; 14 among them grew two bacteria. All patients had Escherichia coli isolate, while Klebsiella pneumoniae was the second commonest bacterium (Table 2 ).

\section{Antimicrobial susceptibility}

Thirty-three isolates $(50.0 \%)$ were resistant to ciprofloxacin (Table 3). The commonest ciprofloxacinresistant bacterium was E. coli (47.0\%). More than $90 \%$ of $E$. coli isolates were sensitive to ampicillin-sulbactam and amoxicillin-clavulanate. Among 9 isolates of $K$. pneumoniae, 8 were sensitive to ampicillin-sulbactam and amoxicillin-clavulanate (Table 4).
Table 1. Characteristics of the study subjects $(n=52)$

\begin{tabular}{|c|c|c|}
\hline Characteristics & $\mathbf{n}$ & $\%$ \\
\hline Age $<60$ years & 8 & 15.4 \\
\hline Urinary catheterization & 6 & 11.5 \\
\hline History of UTI in the last 3 months & 3 & 5.8 \\
\hline \multicolumn{3}{|l|}{ Type of care } \\
\hline In-patient & 2 & 3.8 \\
\hline Out-patient & 50 & 96.2 \\
\hline History of diabetes mellitus & 7 & 13.5 \\
\hline $\begin{array}{l}\text { History of fluoroquinolone } \\
\text { use in the past } 6 \text { months }\end{array}$ & 5 & 9.6 \\
\hline $\begin{array}{l}\text { History of other antibiotic } \\
\text { use in the past } 6 \text { months }\end{array}$ & 3 & 5.8 \\
\hline History of previous prostate biopsy & 2 & 3.8 \\
\hline Histopathological prostatitis & 7 & 13.5 \\
\hline
\end{tabular}

Table 2. Isolated organisms from rectal swabs $(n=66)$

\begin{tabular}{lll}
\hline Organism & $\mathbf{n}$ & \% \\
\hline Escherichia coli & 52 & 78.8 \\
Klebsiella pneumoniae & 9 & 13.6 \\
Enterococcus faecium & 2 & 3.0 \\
Acinetobacter haemolyticus & 1 & 1.5 \\
Morganella morganii subsp. morganii & 1 & 1.5 \\
Enterococcus faecalis & 1 & 1.5 \\
\hline
\end{tabular}

*sum of percentage may not be $100 \%$ due to rounding

Table 3. Bacterial susceptibility against ciprofloxacin

\begin{tabular}{lllll}
\hline Bacteria & $\mathbf{n}$ & Susceptible & Intermediate & Resistant \\
\hline Escherichia coli & 52 & $19(36.5 \%)$ & $2(3.8 \%)$ & $31(59.7 \%)$ \\
Klebsiella pneumoniae & 9 & $7(77.7 \%)$ & $2(22.3 \%)$ & - \\
Enterococcus faecium & 2 & - & 2 & - \\
Acinetobacter haemolyticus & 1 & 1 & - & 1 \\
Morganella morganii subsp. morganii & 1 & - & - & 1 \\
Enterococcus faecalis & 1 & - & & - \\
\hline
\end{tabular}

Table 4. Bacterial susceptibility against ciprofloxacin, ampicillin sulbactam and amoxicillin-clavulanate

\begin{tabular}{llccc}
\hline Bacteria & $\mathbf{n}$ & Ciprofloxacin & Ampicillin sulbactam & Amoxicillin clavulanate \\
\hline Escherichia coli & 52 & $19(36.5 \%)$ & $50(96.2 \%)$ & $47(90.4 \%)$ \\
Klebsiella pneumoniae & 9 & $7(77.7 \%)$ & $8(88.9 \%)$ & $8(88.9 \%)$ \\
Enterococcus faecium & 2 & $0(0 \%)$ & $2(100 \%)$ & $2(100 \%)$ \\
Acinetobacter haemolyticus & 1 & $1(100 \%)$ & $1(100 \%)$ & $1(100 \%)$ \\
Morganella morganii subsp. morganii & 1 & $0(0 \%)$ & $1(100 \%)$ & $1(100 \%)$ \\
Enterococcus faecalis & 1 & $0(0 \%)$ & $1(100 \%)$ & $1(100 \%)$ \\
\hline
\end{tabular}


The E. coli antibiogram showed high resistance to fluoroquinolones $(59.6 \%$ to ciprofloxacin, $57.7 \%$ to levofloxacin) and to third-generation cephalosporins (34.6\% to cefoperazone, $32.7 \%$ to ceftriaxone, and $30.8 \%$ to cefotaxime). All of $E$. coli isolates were susceptible to meropenem or ertapenem and showed very high susceptibility to imipenem (98.1\%), doripenem (98.1\%), piperacillin-tazobactam (96.2\%), and ampicillin-sulbactam (96.2\%) (Table 5).

All isolates of $K$. pneumoniae showed susceptibility to levofloxacin, amikacin, imipenem, doripenem, meropenem, and ertapenem (Table 6). The isolate of Acinetobacter haemolyticus showed no resistance to all antibiotics tested and intermediate resistance to cefoperazone. The isolate of Morganella morganii subsp. morganii showed resistance to ciprofloxacin, cephalothin, amoxicillin-clavulanate, and intermediate resistance to levofloxacin.

Only seven antibiotics were tested to the Enterococci. The two isolates of Enterococcus faecium showed intermediate resistance to ciprofloxacin but were sensitive to ampicillin, teicoplanin, vancomycin, and fosfomycin. One isolate was resistant to linezolid and another one had intermediate resistance to levofloxacin.

Table 5. Antibiogram of Escherichia coli $(n=52)$

\begin{tabular}{|c|c|c|c|}
\hline Antibiotic & Susceptible & Intermediate & Resistant \\
\hline Ciprofloxacin & $19(36.5 \%)$ & 2 (3.8 \%) & $31(59.6 \%)$ \\
\hline Levofloxacin & $20(38.5 \%)$ & 2 (3.8 \%) & $30(57.7 \%)$ \\
\hline Gentamicin & $46(88.5 \%)$ & 0 & 6 (12.8 \%) \\
\hline Amikacin & 47 (90.4 \%) & 5 (9.6 \%) & 0 \\
\hline $\begin{array}{l}\text { Ampicillin- } \\
\text { sulbactam }\end{array}$ & 50 (96.2 \%) & $2(3.8 \%)$ & 0 \\
\hline Cephalothin & 11 (21.2 \%) & $18(34.6 \%)$ & 23 (44.2\%) \\
\hline Cefotaxime & $32(61.5 \%)$ & $4(7.7 \%)$ & $16(30.8 \%)$ \\
\hline $\begin{array}{l}\text { Amoxicillin- } \\
\text { clavulanate }\end{array}$ & 47 (90.4 \%) & 4 (7.7 \%) & 1 (1.9 \%) \\
\hline Ceftriaxone & $34(65.4 \%)$ & 1 (1.9 \%) & 17 (32.7 \%) \\
\hline Ceftazidime & $44(84.6 \%)$ & $3(5.8 \%)$ & 5 (9.6 \%) \\
\hline Cefoperazone & $29(55.8 \%)$ & $5(9.6 \%)$ & 18 (34.6\%) \\
\hline $\begin{array}{l}\text { Piperacillin- } \\
\text { tazobactam }\end{array}$ & $50(96.2 \%)$ & $2(3.8 \%)$ & 0 \\
\hline Cefepime & $43(82.7 \%)$ & 7 (13.5 \%) & 2 (3.8 \%) \\
\hline Imipenem & 51 (98.1 \%) & 1 (1.9 \%) & 0 \\
\hline Doripenem & 51 (98.1 \%) & 0 & 1 (1.9\%) \\
\hline Meropenem & 52 (100\%) & 0 & 0 \\
\hline Ertapenem & 52 (100\%) & 0 & 0 \\
\hline
\end{tabular}

The isolate of Enterococcus faecalis showed resistance to ciprofloxacin and levofloxacin. This isolate was sensitive to ampicillin, teicoplanin, linezolid, vancomycin, and fosfomycin.

Resistant E. coli isolates were of special concern. From the 31 resistant isolates, none was sensitive to levofloxacin, but all were sensitive to carbapenems. Additionally, these isolates showed high susceptibility to amikacin (93.5\%), ampicillin-sulbactam (93.5\%), piperacillin-tazobactam (93.5\%), amoxicillin-clavulanate $(83.9 \%)$, and gentamicin (80.6\%). However, sensitivity to other antibiotics was much higher in ciprofloxacinsensitive $E$. coli isolates. The use of fluoroquinolone in the past six months tended to be associated with ciprofloxacin resistance (Table 7 ).

\section{Post-biopsy infection}

Post-biopsy fever was observed in 7 (13.5\%) patients; 5 of them had resistant isolate to ciprofloxacin. No clinical factor was associated with infection. However, a history of fluoroquinolone treatment in the past 6 months tended to increase the risk of post-biopsy infection (Table 8).

Table 6. Antibiogram of Klebsiella pneumoniae $(n=9)$

\begin{tabular}{|c|c|c|c|}
\hline Antibiotic & Susceptible & Intermediate & Resistant \\
\hline Ciprofloxacin & 7 (77.7 \%) & $2(22.3 \%)$ & 0 \\
\hline Levofloxacin & 9 (100 \%) & 0 & 0 \\
\hline Gentamicin & $8(88.9 \%)$ & 0 & 1 (11.1\%) \\
\hline Amikacin & 9 (100 \%) & 0 & 0 \\
\hline $\begin{array}{l}\text { Ampicillin- } \\
\text { sulbactam }\end{array}$ & 8 (88.9 \%) & 0 & 1 (11.1 \%) \\
\hline Cephalothin & 7 (77.7 \%) & 0 & 2 (22.3\%) \\
\hline Cefotaxime & $6(66.6 \%)$ & 1 (11.1\%) & 2 (22.3\%) \\
\hline $\begin{array}{l}\text { Amoxicillin- } \\
\text { clavulanate }\end{array}$ & 8 (88.9 \%) & 1 (11.1\%) & 0 \\
\hline Ceftriaxone & 7 (77.7 \%) & 1 (11.1 \%) & 1 (11.1 \%) \\
\hline Ceftazidime & 7 (77.7 \%) & 1 (11.1\%) & 1 (11.1 \%) \\
\hline Cefoperazone & 7 (77.7 \%) & 0 & 2 (22.3\%) \\
\hline $\begin{array}{l}\text { Piperacillin- } \\
\text { tazobactam }\end{array}$ & 7 (77.7 \%) & $2(22.3 \%)$ & 0 \\
\hline Cefepime & 7 (77.7 \%) & 1 (11.1 \%) & 1 (11.1\%) \\
\hline Imipenem & 9 (100 \%) & 0 & 0 \\
\hline Doripenem & 9 (100 \%) & 0 & 0 \\
\hline Meropenem & 9 (100\%) & 0 & 0 \\
\hline Ertapenem & 9 (100\%) & 0 & 0 \\
\hline
\end{tabular}


Table 7. Clinical factors associated with ciprofloxacin-resistant isolates

\begin{tabular}{|c|c|c|c|}
\hline \multirow{2}{*}{ Variables } & \multicolumn{2}{|c|}{ Ciprofloxacin } & \multirow{2}{*}{$P$ value $^{1}$} \\
\hline & $R(n=31)$ & $S(n=21)$ & \\
\hline \multicolumn{4}{|c|}{ Current urinary catheterization } \\
\hline Yes & $4(12.9 \%)$ & $2(9.5 \%)$ & 1.000 \\
\hline No & $27(87.1 \%)$ & $19(90.5 \%)$ & \\
\hline \multicolumn{4}{|c|}{ History of UTI in the past 3 months } \\
\hline Yes & $3(9.7 \%)$ & 0 & 0.264 \\
\hline No & $28(90.3 \%)$ & $21(100 \%)$ & \\
\hline \multicolumn{4}{|c|}{ Type of hospital care } \\
\hline In-patient & $2(6.5 \%)$ & 0 & 0.509 \\
\hline Out-patient & $29(93.5 \%)$ & $21(100 \%)$ & \\
\hline \multicolumn{4}{|c|}{ Presence of diabetes mellitus } \\
\hline Yes & $4(12.9 \%)$ & $3(14.3 \%)$ & 1.000 \\
\hline No & $27(87.1 \%)$ & $18(85.7 \%)$ & \\
\hline \multicolumn{4}{|c|}{ Fluoroquinolone use in the past 6 months } \\
\hline Yes & $6(19.4 \%)$ & 0 & 0.070 \\
\hline No & $25(80.6 \%)$ & $21(100 \%)$ & \\
\hline \multicolumn{4}{|c|}{ Other antibiotics in the past 6 months } \\
\hline Yes & $3(9.7 \%)$ & 0 & 0.264 \\
\hline No & $28(90.3 \%)$ & $21(100 \%)$ & \\
\hline \multicolumn{4}{|c|}{ Previous prostate biopsy } \\
\hline Yes & $2(6.5 \%)$ & 0 & 0.509 \\
\hline No & $29(93.5 \%)$ & $21(100 \%)$ & \\
\hline \multicolumn{4}{|c|}{ Histopathological prostatitis } \\
\hline Yes & $5(16.1 \%)$ & $2(9.5 \%)$ & 0.687 \\
\hline No & 26 (83.9\%) & 19 (90.5\%) & \\
\hline
\end{tabular}

${ }^{1}$ Fisher's exact test

Table 8. Factors associated with post-biopsy infection

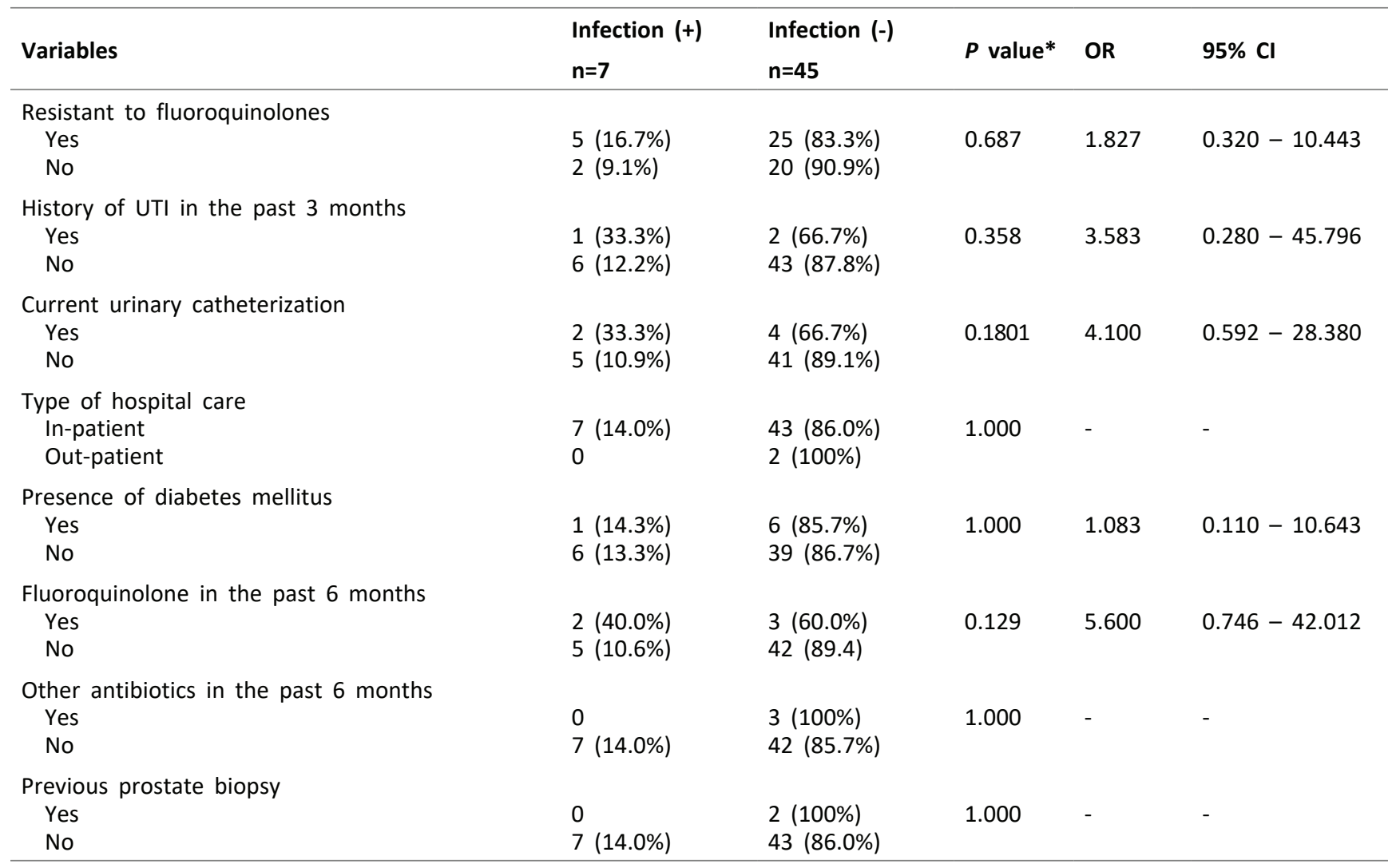

*Fisher's exact test 


\section{DISCUSSION}

This is the first study in Indonesia to evaluate the resistant pattern of bacterial isolates in the rectum of male patients prior to TRUS-guided prostate biopsy. In this current study, E. coli was the most frequently found rectal flora (78.8\%). A similar study in Hong Kong found that $E$. coli was the commonest isolated organism (89.8\%) [13]. While in Korea, E. coli was found in $76 \%$ of cases with positive rectal swabs [15]. A US study in 1,274 patients found $31(2.4 \%)$ cases presented with post-biopsy infections. Positive cultures from blood or urine showed $89 \%$ among the isolates were E. coli bacteria and $90 \%$ of them were fluoroquinolone-resistant [7]. Another study found that E. coli was the commonest pathogen (75\%) in cases with post-biopsy infections [16].

The proportion of fluoroquinolone-resistant bacteria in this study was high (50.0\% for ciprofloxacin), which was mostly predominated by $E$. coli. As a comparison, previous studies in developed countries found a prevalence of $22 \%$ in the United States [17], $10.6 \%$ in London [9], 19\% in Canada [18], and 26.7\% in Korea [15].

Uncontrolled use of fluoroquinolone in society could be responsible for the high bacterial resistance to it. In many cities in Indonesia, especially Jakarta, antibiotics can be purchased without prescriptions such as from "drug stores" which usually act as "peoples' pharmacy". The availability of over-the-counter antibiotics without prescriptions is found not only in Indonesia but also in other developing countries such as Bangladesh, the Philippines, and Mexico. One contributing factor to it is poverty, i.e. when people cannot get free or cheap health treatment in primary health care, they try to get cheaper costs by getting antibiotics and other drugs over the counter. Self-antibiotic treatment usually led to under dosage and inadequate duration of antibiotic treatment which then contributes to the emergence of multi-drug resistant bacteria.

Another contributing factor is the misuse of antibiotics (including ciprofloxacin) as a "growth promoter" in animal food, especially in poultry. It induced the Enterobacteriaceae in the animal gut, especially Escherichia coli and Klebsiella pneumoniae to become resistant to ciprofloxacin. This ciprofloxacin-resistant Escherichia coli and Klebsiella pneumoniae could contaminate the raw food material and the cooked food, which is then consumed by people and then becomes the normal flora in human guts. The Indonesian government has already banned the misuse of antibiotics in animal food.

The other contributing factor is ciprofloxacin and levofloxacin (quinolones) are relatively "new" compared to the "old" antibiotics such as amoxicillin-clavulanate and ampicillin-sulbactam which have been available since 40-50 years ago. Therefore, many physicians tend to
In this study, no clinical factor was found to be associated with fluoroquinolone-resistant $E$. coli. The small number of patients with certain clinical characteristics might affect statistical analyses and significance. For example, only two patients had a history of prostate biopsy before and only five patients received fluoroquinolone in the past six months.

A study in Hong Kong found that diabetes mellitus and prior antibiotics within the last five years are significant predictors for fluoroquinolone-resistant bacteria found in rectal swab cultures [14]. Potential risk factors for fluoroquinolone-resistant are hospitalization, diabetes, and prior treatment with fluoroquinolone [3]. The American Urological Association has also stated that the commonest risk factor for fluoroquinolone-resistant bacteria among patients undergoing prostate biopsy is fluoroquinolone exposure during the last 6 months [10]. Besides fluoroquinolone, the extended-spectrum betalactamase (ESBL)-producing bacteria have also raised awareness. Resistant $E$. coli to the third-generation cephalosporin in this study was also high (above 30\%). A study in the US found only $0.64 \%$ incidence of ESBL producing bacteria [19]. The reported incidence of ESBLproducing organisms is $0.8 \%-1.3 \%$ [20].

Despite the high proportion of bacterial resistance in this study, infectious complication after the procedure was relatively low (13.5\%). However, the reported incidence of UTI after TRUS-guided prostate biopsy was $2 \%-6 \%[5,6]$. Post-biopsy infections by the ESBL-organism have also been reported and may be co-resistant to fluoroquinolone as well $[3,21,22]$. Generally, the pathogens are resistant to third-generation cephalosporins but sensitive to carbapenems [23]. A similar pattern was also seen in this study; almost all $E$. coli isolates were sensitive to carbapenems. Of interest, high susceptibility was also observed with ampicillinsulbactam, piperacillin-tazobactam, and amoxicillinclavulanate. In contrast, a previous study in the US found that fluoroquinolone-resistant E. coli showed $94 \%$ resistance to ampicillin and $74 \%$ resistance to ampicillinsulbactam [17]. Another study in Hong Kong found that rectal bacterial isolates showed high resistance to ampicillin (93.6\%) and ciprofloxacin (60.7\%), but $96.7 \%$ were sensitive to amoxicillin-clavulanate and $100 \%$ sensitive to piperacillin-tazobactam [14]. Our finding is highly suggestive of using ampicillin-sulbactam and amoxicillin-clavulanate as alternative options for postbiopsy infections resistant to fluoroquinolone. In comparison, a randomized trial in patients with complicated skin and skin structure infections found that tigecycline treatment was equally effective compared to ampicillin-sulbactam or amoxicillinclavulanate [24]. This older antibiotic combination seems promising as an alternative antimicrobial treatment in patients with fluoroquinolone resistance. 
We did not find any association between the clinical variable tested and post-biopsy infection. This result could be due to the small sample size and low incidence of risk factors, such as a history of UTI in the last 3 months, urinary catheterization, hospitalization, diabetes mellitus, and prior prostate biopsy. However, the use of fluoroquinolone in the past six months may contribute to the present infection. Our results indicated that all six patients with a history of fluoroquinolone use also showed ciprofloxacin resistance. The statistic did not reach significance due to the small sample size of patients with a history of fluoroquinolone use and zero result in the patient's subgroup without ciprofloxacin resistance.

In comparison, a study in Pakistan involving 158 patients undergoing TRUS-guided biopsy found an infection rate of $12.5 \%$. The rate was significantly higher among patients carrying fluoroquinolone-resistant bacteria than non-carriers ( $24 \%$ vs. $3.5 \%, P<.001)$. Previous use of fluoroquinolone (OR, 2.54;95\% Cl, 1.17-5.49; $P=$ .019) and history of hospitalization (OR, 7.85; $95 \% \mathrm{Cl}$, 2.075-29.744; $P=.002$ ) were identified as independent risk factors of fluoroquinolone resistance [25]. This study strongly emphasizes the need for appropriate prophylaxis to reduce the post-biopsy infection rate.

Modification of prophylaxis antibiotics might be needed to reduce infectious complications. In Korea, for instance, the previous policy was to give ciprofloxacin $500 \mathrm{mg}$ before 2009, which resulted in an increase in the incidence rate of post-biopsy infection from $0.3 \%$ to $2.31 \%$. The addition of intravenous ceftriaxone before biopsy plus fluoroquinolone for more than 7 days after biopsy reduced the infection rate to $0.2 \%$ in $2010-2012$ [26]. An alternative regimen with piperacillin-tazobactam 4.5 gram 30 minutes before and 6 hours after TRUSguided biopsy has been proposed in Japan. This regimen showed a similar incidence rate of acute prostatitis with previous fluoro-quinolone-based regimens [27].

Recently, a pre-biopsy screening to identify fluoroquinolone-resistant bacteria using ciprofloxacinsupplemented MacConkey agar has been proposed. Antibiotic prophylaxis was modified in patients showing positive resistant bacteria. This method resulted in reduced post-biopsy sepsis compared to standard empirical antibiotic prophylaxis, but it did not reach statistical significance $(0.66 \%$ vs. $4.3 \% ; P=.08)$. The alternative antibiotic regimen used in this study was trimethoprim-sulfamethoxazole or cefuroxime depending on their resistance profiles [28].

This study has several limitations. The total sample size was 52 patients, but, with the proportion of ciprofloxacin-resistant bacteria as high as $50 \%$, this result was quite significant for considering alternative antimicrobial prophylaxis in the future. Another limitation of this study was the lack of identification of uropathogenic Escherichia coli.

\section{CONCLUSIONS}

Ciprofloxacin-resistant bacteria in the rectum are common among patients undergoing prostate biopsy in Cipto Mangunkusumo Hospital. The proportion is found to be as high as $50.0 \%$ with Escherichia coli as the commonest resistant pathogen. Alternative antimicrobial prophylaxis should be considered in the future; the recommended choices based on the antibiogram profile in this study are ampicillin-sulbactam and amoxicillinclavulanate. Routine pre-biopsy rectal swab cultures should also be encouraged, both to guide prophylaxis and to plan treatment to reduce the incidence of postbiopsy bacteremia and sepsis.

\section{DECLARATIONS}

\section{Competing of Interest}

The authors declare no potential conflicts of interest.

\section{Acknowledgment}

Not applicable

\section{REFERENCES}

1. Liss MA, Peeples AN, Peterson EM. Detection of fluoroquinolone-resistant organisms from rectal swabs by use of selective media prior to a transrectal prostate biopsy. J Clin Microbiol. 2011;49(3):1116-8.

2. Loeb S, Carter HB, Berndt $\mathrm{SI}$, et al. Complications after prostate biopsy: data from SEER-Medicare. J Urol. 2011;186(5):1830-4.

3. Williamson DA, Barrett LK, Rogers BA, et al. Infectious complications following transrectal ultrasound-guided prostate biopsy: new challenges in the era of multidrug-resistant Escherichia coli. Clin Infect Dis. 2013;57(2):267-74.

4. Ozden E, Bostanci Y, Yakupoglu KY, et al. Incidence of acute prostatitis caused by extended-spectrum betalactamase-producing Escherichia coli after transrectal prostate biopsy. Urology. 2009;74(1):119-23.

5. Otrock ZK, Oghlakian GO, Salamoun MM, et al. Incidence of urinary tract infection following transrectal ultrasound guided prostate biopsy at a tertiary-care medical center in Lebanon. Infect Control Hosp Epidemiol. 2004;25(10):873-7.

6. Zaytoun OM, Vargo EH, Rajan R, et al. Emergence of fluoroquinolone-resistant Escherichia coli as cause of postprostate biopsy infection: implications for prophylaxis and treatment. Urology. 2011;77(5):1035-41. 
7. Duplessis CA, Bavaro M, Simons MP, et al. Rectal cultures before transrectal ultrasound-guided prostate biopsy reduce post-prostatic biopsy infection rates. Urology. 2012;79(3):556-61.

8. Feliciano J, Teper E, Ferrandino $M$, et al. The incidence of fluoroquinolone resistant infections after prostate biopsy--are fluoroquinolones still effective prophylaxis? J Urol. 2008;179(3):952-5; discussion 5.

9. Batura D, Gopal Rao G. The national burden of infections after prostate biopsy in England and Wales: a wake-up call for better prevention. J Antimicrob Chemother. 2013;68(2):247-9.

10. European Association of Urology. Guidelines on urological infections. 2015 [cited 2019 6/20]. Available from: https://uroweb.org/wp-content/ uploads/19-Urological-infections_LR2.pdf.

11. American Association of Urology. Best ractice policy statement on urologic surgery antimicrobial prophylaxis. 2012 [cited 2019 06/24]. Available from: https://http://www.auanet.org/guidelines/archiveddocuments/antimicrobial-prophylaxis-best-practicestatement.

12. Drusano GL, Preston SL, Van Guilder $M$, et al. A population pharmacokinetic analysis of the penetration of the prostate by levofloxacin. Antimicrob Agents Chemother. 2000;44(8):2046-51.

13. Lwanga SK, Lemeshow S. Sample Size Determination in Health Studies. A Practical Manual. Geneva: World Health Organization, 1991.

14. Tsu JH, Ma WK, Chan WK, et al. Prevalence and predictive factors of harboring fluoroquinoloneresistant and extended-spectrum $\beta$-lactamaseproducing rectal flora in Hong Kong Chinese men undergoing transrectal ultrasound-guided prostate biopsy. Urology. 2015;85(1):15-21.

15. Lee JW, Park SC, Kim MK, et al. Prevalence of antimicrobial resistance in normal rectal flora of patients undergoing transrectal ultrasonographyguided prostate biopsy in Korea. Int J Urol. 2014;21(8):811-4.

16. Carignan A, Roussy JF, Lapointe V, et al. Increasing risk of infectious complications after transrectal ultrasound-guided prostate biopsies: time to reassess antimicrobial prophylaxis? Eur Urol. 2012;62(3):453-9.

17. Liss MA, Chang A, Santos $R$, et al. Prevalence and significance of fluoroquinolone resistant Escherichia coli in patients undergoing transrectal ultrasound guided prostate needle biopsy. J Urol. 2011;185(4):1283-8.

18. Taylor S, Margolick J, Abughosh Z, et al. Ciprofloxacin resistance in the faecal carriage of patients undergoing transrectal ultrasound guided prostate biopsy. BJU Int. 2013;111(6):946-53.
19. Dai J, Leone A, Mermel L, et al. Rectal swab culturedirected antimicrobial prophylaxis for prostate biopsy and risk of postprocedure infection: a cohort study. Urology. 2015;85(1):8-14.

20. Taylor AK, Zembower TR, Nadler RB, et al. Targeted antimicrobial prophylaxis using rectal swab cultures in men undergoing transrectal ultrasound guided prostate biopsy is associated with reduced incidence of postoperative infectious complications and cost of care. J Urol. 2012;187(4):1275-9.

21. Cannon GM, Jr., Smaldone MC, Paterson DL. Extended-spectrum beta-lactamase gram-negative sepsis following prostate biopsy: implications for use of fluoroquinolone prophylaxis. Can J Urol. 2007;14(4):3653-5.

22. Horcajada JP, Busto M, Grau S, et al. High prevalence of extended-spectrum beta-lactamase-producing enterobacteriaceae in bacteremia after transrectal ultrasound-guided prostate biopsy: a need for changing preventive protocol. Urology. 2009;74(6):1195-9.

23. Pitout JD, Laupland KB. Extended-spectrum betalactamase-producing Enterobacteriaceae: an emerging public-health concern. Lancet Infect Dis. 2008;8(3):159-66.

24. Matthews $P$, Alpert $M$, Rahav G, et al. A randomized trial of tigecycline versus ampicillin-sulbactam or amoxicillin-clavulanate for the treatment of complicated skin and skin structure infections. BMC Infect Dis. 2012;12:297.

25. Hasanzadeh A, Pourmand MR, Alizadeh A, Pourmand $G$. Prevalence and significance of fluoroquinoloneresistant bacteria carriage in patients undergoing transrectal ultrasound prostate biopsy. Urol J. 2017;14(3):3085-90.

26. Lee C, You D, Jeong IG, et al. Antibiotic prophylaxis with intravenous ceftriaxone and fluoroquinolone reduces infectious complications after transrectal ultrasound-guided prostatic biopsy. Korean J Urol. 2015;56(6):466-72.

27. Iwamoto $H$, Shigehara K, Miyagi T, et al. Efficacy of two-time prophylactic intravenous administration of tazobactam/piperacillin for transrectal ultrasoundguided needle biopsy of the prostate. Prostate Int. 2015;3(3):103-6.

28. Farrell JJ, Hicks JL, Wallace SE, Seftel AD. Impact of preoperative screening for rectal colonization with fluoroquinolone-resistant enteric bacteria on the incidence of sepsis following transrectal ultrasound guided prostate biopsy. Res Rep Urol. 2017;9:37-41. 